\title{
STM STUDIES OF THE PROXIMITY EFFECT IN SUPERCONDUCTING WIRES WITH ARTIFICIAL PINNING CENTERS
}

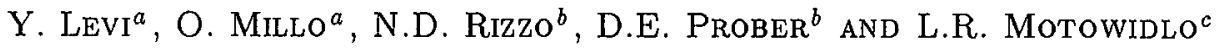 \\ ${ }^{a}$ Racah Institute of Physics, The Hebrew University, Jerusalem 91904, Israel \\ ${ }^{b}$ Applied Physics, Yale University, New Haven, CT 06520-8284, USA \\ ${ }^{c}$ IGC-AS, Waterbury, CT 06704, USA
}

\begin{abstract}
Scanning tunneling microscopy and spectroscopy were applied to study the proximity effect with nanometer spatial resolution. The measurements were conducted on novel superconducting wires, which consist of an ordered array of submicron diameter normal metal filaments ( $\mathrm{Cu}, \mathrm{Ni}$ ) embedded in a superconducting ( $\mathrm{NbTi}$ ) matrix. Spatially resolved information about the local density of states is obtained by taking $I-V$ curves simultaneously with topographic images. Our data provide direct evidence on the interplay between the normal and superconducting constituents in the vicinity of the interface between them.
\end{abstract}

PACS numbers: $74.50 .+\mathrm{r}, 73.40 . \mathrm{Gk}, 61.16 . \mathrm{Ch}$

The mutual effect of a normal metal (N) in good electrical contact with a superconductor (S), a phenomenon known as the proximity effect (PE), has been studied for over three decades $[1,2]$. A new interest in the subject has arisen lately, because of technological advancements that allow to conduct experiments which reveal novel mesoscopic effects [3-5]. One of the main points of interest is the microscopic variations of the superconducting condensate and the pair potential near the N-S boundary, and the way they influence the local density of states (DOS). Most of the experiments that probe the PE dealt so far with macroscopic properties (resistivity, magnetization, etc.), averaging out local information. Tunneling spectroscopy has also been extensively employed, but using relatively large area tunnel junctions where tunneling was performed perpendicular to the $\mathrm{N}-\mathrm{S}$ interface [2].

Local studies of electronic properties were made possible by the development of scanning tunneling microscopy (STM). Some high spatial resolution tunneling spectroscopy measurements of the PE have already been performed [6-8]. However, in these works tunneling occurred through (or near) very thin $N$ islands deposited on top of a bulk superconductor [6], or in granular samples where adjacent grains 

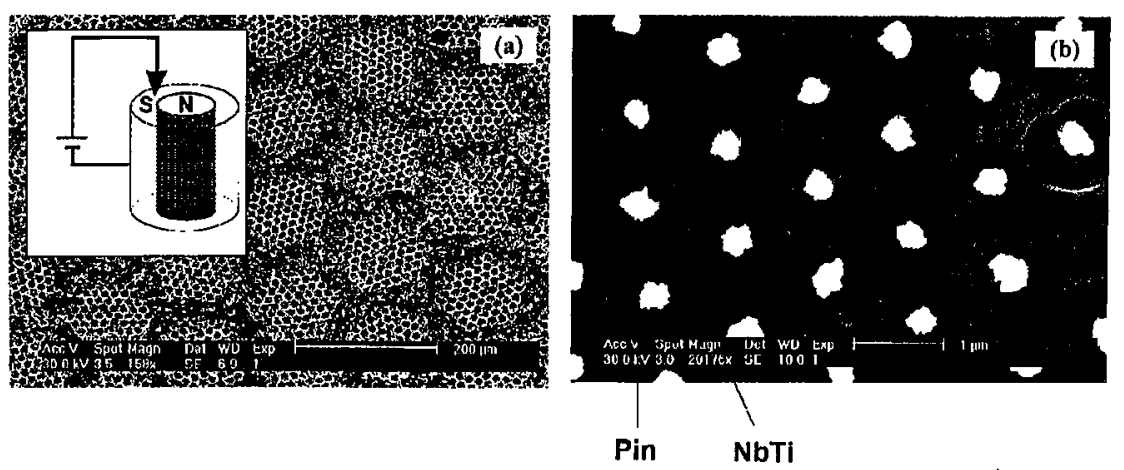

Fig. 1. SEM micrographs of the Ni island-pin wire (\#1). (a) Partial cross-section of the wire displaying a self-similar ordered array of hexagons. Each small hexagon contains an hexagonal array of pins embedded in a superconducting matrix, as can be seen in (b). Insert: schematic of the experimental setup. The arrow represents the STM tip, and tunneling is parallel to the interface between the wire constituents.

were only weakly coupled [8]. As far as we know, this is the first report on spatially resolved measurements where the tunneling current is directed parallel to well defined interfaces between "semi-infinite" slabs of materials in good electrical contact (see inset of Fig. 1).

As opposed to conventional superconducting wires, where flux pins are randomly created, our samples have engineered pins. The artificial pinning center (APC) approach is to embed normal or magnetic materials in a specific ordered filamentary configuration. This idea has already proven itself useful, since wires of this type have higher critical currents (at various magnetic fields) than those manufactured with conventional processing. Details of the fabrication procedures and critical currents vs. fields of these wires are brought elsewhere $[9,10]$. This technological success stimulates further study of the microscopic behavior of these wires, in order to model their PE and continue to optimize them. The submicron diameter of each pin, along with its "semi-infinite" length make it an ideal system for an STM research (performed along the wire's cross-section). Furthermore, good electrical contact between the pins and the superconductor is ensured by the manufacture procedure [3].

We present here the data obtained for two wires, designed and fabricated at IGC-Advanced Superconductors. The first (wire \#1) has the "island-pin" geometry, with a $\mathrm{Ni}$ (ferromagnetic) pin. The "unit filament" consists of a pin placed inside a NbTi (superconductor) filament, whose diameter is reduced after numerous manufacturing steps to $1 \mu \mathrm{m}$. At this stage each pin is made of a cylindrical $\mathrm{Ni}$ filament $200 \mathrm{~nm}$ in diameter, surrounded by a $50 \mathrm{~nm}$ thick $\mathrm{Cu}$ sleeve, that serves as a diffusion barrier against the migration of $\mathrm{Ni}$ into $\mathrm{NbTi}$. The second (wire \#2) has the "barrier-pin" geometry, where at its final stage the "unit filament" is a hexagon of $\mathrm{NbTi} 80 \mathrm{~nm}$ in (diagonal) size, surrounded by a $15 \mathrm{~nm}$ thick $\mathrm{Cu}$ barrier. The pin here is the normal metal wall around the superconductor. Both wires consist of an ordered array of "unit filaments" inside a $\mathrm{Cu}$ cladding. The study of 
these two wires allows to compare a system with a ferromagnetic constituent to a system without magnetic inclusions, and also to see how differences in the lateral dimensions affect the PE.

A few millimeters long pieces were cut from the wire and their cross-section was polished using diamond lapping compounds. Just before mounting them onto our cryogenic STM, the samples were subjected to a short chemical etch that restored their microscopic structure and removed oxides and other surface impurities. Sample micrographs of the Ni island-pin wire are shown in Fig. 1, depicting a self-similar array of hexagons (a), where each small hexagon consists of yet another ordered array of individual "unit filaments", seen only in Fig. 1b.
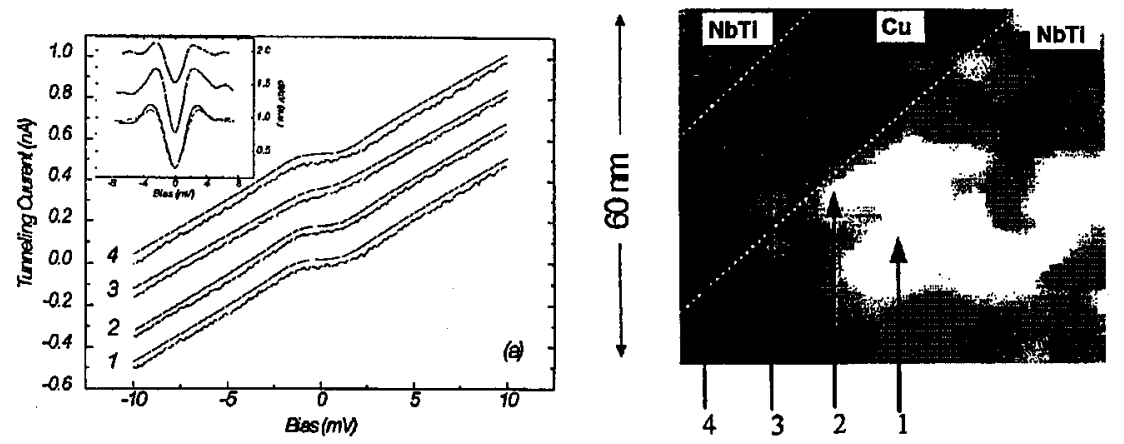

Fig. 2. (a) Four $I-V$ characteristics taken at different lateral tip positions along a $\mathrm{Cu}$ barrier-pin wire (\#2). The smooth lines were calculated as discussed in the text: for curves $1-4, \Delta=1.55,1.45,0.8,1.3 \mathrm{meV}$, respectively. Curves are vertically displaced for clarity. (b) Detail of a STM topographic image of the wire cross-section, showing the locations where the curves in (a) were taken. Curves 1, 2 and 4 are taken on two $\mathrm{NbTi}$ regions that are separated by a $\mathrm{Cu}$ pin, where curve 3 was taken. The boundaries between different materials are designated by dashed lines. Inset: normalized $\mathrm{d} I / \mathrm{d} V$ traces taken deep on NbTi (bottom solid line), at the $\mathrm{NbTi} / \mathrm{Cu}$ interface (middle) and on the $\mathrm{Cu}$ (top). The dotted line is a theoretical fit, with $\Delta=1.55 \mathrm{meV}$.

In order to study the local DOS in correlation with surface structure, we acquired (at $4.2 \mathrm{~K}$ ) topographic images of the wire cross-section simultaneously with $I-V$ characteristics at different lateral tip positions. Particular attention was given to mapping the DOS as a function of the distance from the interface between different materials. In Fig. 2a one can see four experimental $I-V$ curves (fuzzy traces) taken on wire \#2 at different positions, as indicated in the topographic image (Fig. 2b). In this grey scale image, the dark region corresponds to depressed areas while the bright one to elevated ones. The image depicts a small section of a $\mathrm{Cu}$ pin (depressed, due to the different etch rates of the metals) which separates two NbTi regions (lower right and upper left). The variations in the local DOS are manifested in Fig. 2a. Deep in the S side (curve 1) a large spectroscopic gap is apparent, but it diminishes (curve 2) when approaching the $\mathrm{Cu}$. Inside the $\mathrm{Cu}$ (curve 3 ) the gap is swaller but still evident, indicating the effect of supercon- 
ductivity on the normal metal. Finally, when re-entering the NbTi (curve 4) the gap starts to recover. It should be emphasized that both wires were measured also at $\approx 10 \mathrm{~K}$ (just above $T_{\mathrm{C}} \approx 9 \mathrm{~K}$ ) where only gapless curves were found. This indicates that the gaps observed at $4.2 \mathrm{~K}$ are of superconducting origin, and not due to surface impurities, Coulomb charging or other extraneous effects.

The main parameters governing the behavior of the spectra are the width of the gap and their zero bias conductance ( $\mathrm{ZBC}$ ) normalized to the conductance at a high bias. In order to obtain an estimate of the gap we fit the experimental $I-V$ and $\mathrm{d} I / \mathrm{d} V$ vs. $V$ curves using the conventional tunneling expression for $\mathrm{N}$-insulator-S junctions [2]. The local quasiparticle DOS is taken, in a preliminary approach, as $N(E)=(E-\mathrm{i} \Gamma) / \sqrt{(E-\mathrm{i} \Gamma)^{2}-\Delta^{2}}[11] . \Delta$ designates the gap and $\Gamma$ is a phenomenological lifetime broadening. Although this approach is simplistic, and cannot account for all of the details of the $\mathrm{PE}$, it offers a consistent procedure to assess the magnitude of the gap of a given curve. The gap's nature cannot be easily determined, in particular not in $\mathrm{N}$, since it seems too large to be attributed exclusively to a local pair potential. Therefore, it may be also due to a combination of the following: (1) Leakage of Cooper pairs and quasiparticles from $S$ to $N$. (2) A single quasiparticle bound state of energy close to the superconducting gap of $\mathrm{NbTi}[6]$.

Although our theoretical approach gives a rough description of the curves as can be seen in Fig. 2a, some details are missed by this treatment. The major discrepancies are an enhancement of the DOS at the gap edge and some minor peaks above the gap. These may be attributed to quasiparticle bound states, multiple Andreev reflections at the material boundaries and to phonon structure. In the inset of Fig. 2a three experimental $\mathrm{d} I / \mathrm{d} V$ vs. $V$ curves are presented. The bottom one (solid line) was taken deep in the $S$, the middle one was taken on the $\mathrm{NbTi} / \mathrm{Cu}$ boundary and the upper one was taken on the $\mathrm{Cu}$. The dashed line is the theoretical fit for the bottom curve, and indeed is not a perfect fit. These latter issues require a more thorough theoretical treatment $[5,6]$ which will be pursued subsequently. Nevertheless, a general picture may be already obtained.

The variations of the gap, $\Delta$, and the $\mathrm{ZBC}$ as a function of the distance from the boundaries are brought in Figs. $3 \mathrm{a}$ and $\mathrm{b}$ for wires \#1 and \#2 respectively. The values of $\Delta$ are obtained from the fits discussed above. The arrows in the insets illustrate the direction along which the data was acquired, and the materials encountered. One can see (Fig. 3a) that superconductivity does not penetrate the $\mathrm{Ni}$, but traces of it are found in the $\mathrm{Cu}$, adjacent to the $\mathrm{Ni}$. As one draws further away from the pin, $\Delta$ rises and the ZBC drops until saturation is reached 50 to $60 \mathrm{~nm}$ from the $\mathrm{NbTi} / \mathrm{Cu}$ interface: $\Delta=1.55 \mathrm{meV}$ (yielding the established [2] ratio for $\mathrm{NbTi}, 2 \Delta / k_{\mathrm{B}} \approx 4$ ) and $\mathrm{ZBC}=0.15$. In the $\mathrm{Cu}$ barrier-pin wire (Fig. $3 \mathrm{~b}$ ) the healing length, namely the distance from the $\mathrm{NbTi} / \mathrm{Cu}$ boundary where saturation occurs, is shorter, about 30 to $40 \mathrm{~nm}$. Here also superconductivity is monotonically suppressed when approaching the $\mathrm{Cu}$ wall, and reaches a minimum approximately midway between the two $S$ filaments, as expected.

The main conclusions are as follows: (1) No evidence of superconductivity can be found in the Ni. (2) Superconductivity affects the DOS of the $\mathrm{Cu}$, for a distance at least $50 \mathrm{~nm}$ from the $\mathrm{Cu} / \mathrm{NbTi}$ boundary, even in the presence of the $\mathrm{Ni}$ 

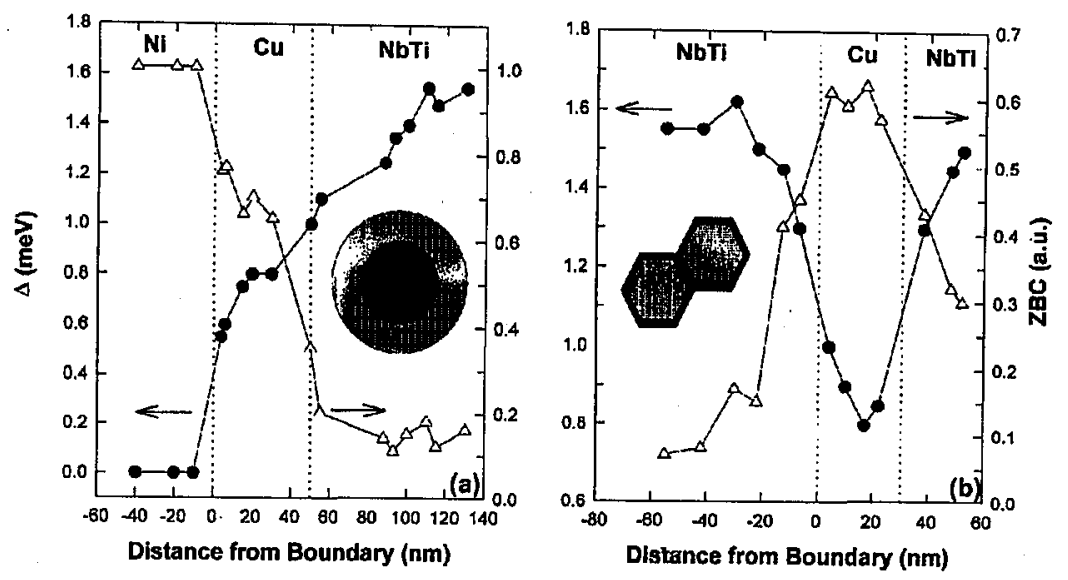

Fig. 3. (a) Spectroscopic gap, $\Delta$ (black circles, left axis) and normalized ZBC (white triangles, right axis) data taken on the $\mathrm{Ni}$ island pin wire (\#1) as a function of distance from the $\mathrm{Ni} / \mathrm{Cu}$ interface. The experimental errors on the data are approximately the size of the symbols. Inset: diagram of the area studied, with an arrow illustrating the scan line on which the $I-V$ curves were taken. (b) Same as (a), but for the Cu barrier-pin wire (\#2). Note the difference in scales.

pin. This is comparable to the result of the dirty limit approximation [2] that yields a penetration distance of 50 to $100 \mathrm{~nm}$ into the $\mathrm{Cu}$. (3) There is a suppression of superconductivity in $\mathrm{S}$ near the $\mathrm{N}-\mathrm{S}$ boundary. The superconductivity healing length is larger in wire \#1 (50 to $60 \mathrm{~nm}$ ) than in wire \#2 (30 to $40 \mathrm{~nm})$. Possible reasons are the thicker $\mathrm{Cu}$ region and the presence of a magnetic material in wire \#1. We would like to point out here that both healing lengths are much longer than the coherence length reported in [12] for NbTi at $4.2 \mathrm{~K}, \xi_{\mathrm{s}} \approx 5 \mathrm{~nm}$. This is probably due to some intermixing of the materials at each interface that occurs during wire manufacture, smearing the boundaries. It may also result from the pronounced $\mathrm{PE}$ induced by the $\mathrm{Cu}$; and in particular the Ni layers. We recall that in previous experiments $[8,13]$ where electrical contact was poor and no intermixing occurred, the healing length in $\mathrm{S}$ was found to be of the order of $\xi_{\mathrm{s}}$, and superconductivity hardly penetrated N. Further investigation is needed in order to clarify this issue as well as the origin of the gap in the normal region.

\section{References}

[1] G. Deutscher, P.G. de Gennes, in: Superconductivity, Vol. 2, Ed. R.D. Parks, Dekker, New York 1969.

[2] E.L. Wolf, Principles of Electron Tunneling Spectroscopy, Oxford University Press, Oxford 1989.

[3] A.C. Mota, P. Visani, A. Pollini, K. Aupke, Physica B 197, 95 (1994).

[4] V. Petrashov, V.N. Antonov, P. Desling, T. Cleason, Phys. Rev. Lett. 74, 5268 (1995); H. Courtois, Ph. Gandit, D. Mailly, B. Panettier, ibid. 76, 130 (1996).

[5] S. Gueron, H. Pothier, N.O. Birge, D. Esteve, M.H. Devoret, Phys. Rev. Lett. 77, 3025 (1996). 
[6] S.H. Tessmer, D.J. Van Harlingen, J.W. Lyding, Phys. Rev. Lett. 70, 3135 (1993); S.H. Tessmer, M.B. Tarlie, D.J. Van Harlingen, D.L. Maslov, P.M. Goldbart, ibid. 77, 924 (1996).

[7] K. Inoue, H. Takayanagi, Phys. Rev. B 43, 6214 (1991).

[8] O. Millo, Y. Levi, E. Bar-Sadeh, U. Asaf, I. Felner, J. Low Temp. Phys, 106, 417 (1997).

[9] L.R. Motowidlo, B.A. Zeitlin, M.S. Walker, P. Haldar, Appl. Phys. Lett. 61, 991 (1992); N.D. Rizzo, J.Q. Wang, D.E. Prober, L.R. Motowidlo, B.A. Zeitlin, ibid. 69, $2285(1996)$.

[10] R.W. Heussner, J.D. Marquandt, P.J. Lee, D.C. Larbalestier, Appl. Phys. Lett. 70, 901 (1997).

[11] R.C. Dynes, V. Narayanamurti, J.P. Garno, Phys. Rev. Lett. 41, 1509 (1978).

[12] X.S. Ling, J.D. McCambridge, N.D. Rizzo, J.W. Sleight, D.E. Prober, L.R. Motowidlo, B.A. Zeitlin, Phys. Rev. Lett. 74, 805 (1995).

[13] E. Bar-Sadeh, I. Felner, U. Asaf, O. Millo, Phys. Rev. B 52, 6734 (1995). 